\title{
Bone formation in rabbit cancellous bone explant culture model is enhanced by mechanical load
}

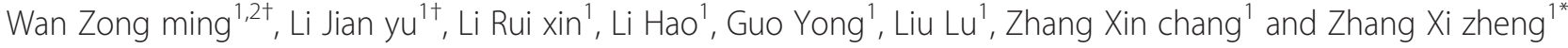

\author{
* Correspondence: z84656716@ \\ yahoo.com \\ ${ }^{\dagger}$ Equal contributors \\ ${ }^{1}$ Institute of Medical Equipment, \\ Academy of Military Medical \\ Sciences, Tianjin, China \\ Full list of author information is \\ available at the end of the article
}

\begin{abstract}
Background: When studying and designing an artificial bone in vitro with similar features and functionality of natural bone by tissue engineering technology, the culturing environment, especially the mechanical environment is supposed to be an important factor, because a suitable mechanical environment in vitro may improve the adaptability of the planted-in tissue engineering bone in the body. Unfortunately, up to now, the relationship between mechanical stimuli and natural bone growth has not yet been precisely determined, and it is so imperative for a prior study on effect of mechanical loading on growth of the natural bone cultured in vitro.

Methods: Under sterile conditions, explant models of rabbit cancellous bone with $3 \mathrm{~mm}$ in thickness and $8 \mathrm{~mm}$ in diameter were prepared and cultured in a dynamic loading and circulating perfusion bioreactor system. By Micro-CT scanning, a 3D model for finite element (FEM) analysis was achieved. According to the results of FEM analysis and physiological load bearing capacity of the natural bone, these models were firstly subjected to mechanical load with $1 \mathrm{~Hz}$ frequency causing average apparent strain of $1000 \mu \varepsilon, 2000 \mu \varepsilon, 3000 \mu \varepsilon$ and $4000 \mu \varepsilon$ respectively for 30 min every day, activities of alkaline phosphatase (AKP) were detected on the $5^{\text {th }}$ and the $14^{\text {th }}$ loading day and on the $14^{\text {th }}$ and the $21^{\text {st }}$ day, mechanical properties, tissue mineral density (TMD) of the bone explant models were investigated and Von-kossa staining and fluorescence double labeling assays were conducted to evaluate whether there were fresh osteoid in the bone explant models. In addition, Western blot, Elisa and Real-time PCR were employed to analyze expression of Collagen-I (COL-1), bone morphogenetic protein-2 (BMP-2) and osteoprotegerin (OPG) protein and RNA.

Results: The explant models of rabbit cancellous bone prepared under sterile conditions grew well in the bioreactor system. With the increasing culturing time and load levels, bone explant models in groups with $1000 \mu \varepsilon$ and $2000 \mu \varepsilon$ average apparent strain experienced improving mechanical properties and TMD $(P<0.05)$, and results of Von-kossa staining and fluorescence double labeling also showed apparent fresh osteoid formation. Under the same loading conditions, a up-regulations in protein and RNA of COL-1, BMP-2 and OPG were detected, especially, relative genes notably expressed after 21 days.
\end{abstract}

Conclusion: Our study demonstrated that mechanical load could improve function and activity of osteoblasts in explant models of cancellous bone. Through regulations of COL-1, OPG and BMP-2 secreted by osteoblasts, the mechanical load could improve the tissue structural density and stiffness due to formation of fresh osteoid.

Keywords: Bone tissue engineering, Mechanical load, Bone explant culture, Osteoblast

\section{() Biomed Central}

(c) 2013 Zong ming et al.; licensee BioMed Central Ltd. This is an Open Access article distributed under the terms of the Creative Commons Attribution License (http://creativecommons.org/licenses/by/2.0), which permits unrestricted use, distribution, and reproduction in any medium, provided the original work is properly cited. 


\section{Introduction}

Natural bone formation in vivo is a complex process in which involved contribution of multiple cell types, physical and biological environment [1]. Mechanical cues play an important role in bone regeneration and affect production and secretion dynamics of growth factors (GFs) involved in osteogenesis [2-5]. In the 19th century, Julius Wolff firstly suggested that external mechanical load can effectively change bone shape and structure [6]. In 1987, Frost raised the "mechanostat" theory which has made a better explanation to Wolff's law in the level of tissue $[7,8]$. The positive influences of mechanical load on bone metabolism with improved bone healing or remodeling have been clearly demonstrated in the veterinary and clinical setting. However the processes involved in mechanical signaling remain in the most part obscure.

Previous work has investigated some mechano-responsiveness of involvement of GFs in osteogenesis with two-dimensional monolayer cell culture models in vitro. Using a fourpoint bending device, MC3T3-E1 cells (a mouse monoclonal pre- osteoblastic cell line) were exposed to mechanical tensile strain, which resulted in the altered expression of 1992 genes, 41 of which were involved in the mitogen-activated protein kinase (MAPK) signaling pathway, ERK in addition, also played an important role in response to mechanical strain, while the membrane-associated receptors integrin $\beta 1$ and $\beta 5$ were determined to regulate ERK activity and proliferation of cells in opposite ways. Mechanical tensile strain could also apparently promote osteoblasts differentiation through BMPs/Smad pathway in vitro, in turn, it could lead to accumulation of Smad proteins caused by a drop in Smurf levels, subsequently, and enhance BMPs/Smad signaling [9,10], but they did not effectively embody the physiological interactions either between neighboring cells of different types or between cells and extracellular matrix. However, bone tissue contains a large number of different cell types which interact to maintain the bone metabolism. Some studies have confirmed that in bone, mechanical stimuli is transmitted through the extracellular matrix (ECM) to resident osteoblasts, osteocytes, periosteal cells and osteoclasts [11,12], therefore, there is a need for models in vitro that represent the physiological diversity and characteristics of bone formation to practically study the effects of mechanical cues on this process.

Bone explant culture has a short lifespan in vitro, as they often undergo central necrosis due to vascular occlusion and rate-limiting mass transfer. The loss of the vascular system has implications in limiting the size of tissue sample that can be harvested, since cells in culture depend upon diffusion of nutrients and metabolites as well as for removal of waste by vascular system. Proliferation may thus be limited to the outer cell layer while necrosis may occur in the centre of the explants. Jones et al. [13] designed an ex vivo mechanical load culture system for 3D ovine, bovine and human cancellous tissue which overcame some of the limitations discussed above. There is currently a great deal of interest in trying to develop artificial bone in vitro by tissue engineering, Jaasma et al. [14] developed a dynamic flow perfusion bioreactor which led to a increase in early-stage bone formation marker of collagen-GAG scaffolds seeded with osteoblasts.

In this study, we used a new dynamic load and circulating perfusion bioreactor system which was independently developed by Academy of Military Medical Science, China [15]. It could accurately provide a compressive strain with different magnitudes and frequencies, as well as perfusions under different flow conditions with easy control and steady performance, which could be an ideal dynamic culture and loading device for cultivation of natural bone and tissue engineering bone. 
The aim of the present study was to determine whether the rabbit cancellous bone explant models responded with physiological reaction patterns to force. The physiological reaction patterns were reflected by the increase in apparent stiffness and bone mass in the form of newly-formed osteoid. In that way, we will demonstrate the growing microenvironment of tissue engineering bone in vitro.

\section{Materials and methods}

\section{Materials}

Dulbecco's modified Eagle's medium (DMEM) with Penicillin $100 \mathrm{U} / \mathrm{ml}$ and Streptomycin $100 \mu \mathrm{g} / \mathrm{ml}$ and Fetal bovine serum (FBS) were obtained from HyClone, USA. Protein Quantification Kit and Alkaline Phosphatase (AKP) Delection Kit were manufactured by Nanjing Jiancheng Bioengineering Institute, China. Tetracycline hydrochloride (\#0422) was purchased from Amresco (Amresco, USA), Calcein (\#0875) was purchased from Sigma (Sigma, USA), Mouse Anti-Collagen I antibody [COL-1] (\#ab90395) was purchased from Abcam (HK) Ltd., Rabbit Osteoprotegerin (OPG) ELISA Kit and Rabbit Bone Morphogenetic Protein-2 (Bmp-2) ELISA Kit were purchased from Cusabio Biotech Co., LTD, USA. Von-kossa Ca Staining Kit was purchased from GENMED SCIENTIFICS INC., USA. TRIZOL was purchased from Invitrogen (Invitrogen, USA). All other chemicals of reagent grades were obtained from Sigma unless otherwise noted.

\section{Animals}

Naturally mated 3-month old New Zealand White rabbits were obtained from the Laboratory Animal Center of Academy of Military Medical Sciences, China. The animal experiments were in accordance with the governmental guidelines for the care and use of laboratory animals and approved by Academy of Military Medical Sciences Ethics Committee, China.

\section{Preparation of rabbit cancellous bone explant model}

Firstly, rabbit femoral heads were extracted from two legs of 3 month-old rabbit, then a femoral head was merely made into one cancellous bone tissue slice with $3 \mathrm{~mm}$ thickness in an aseptic processing cutting machine which was designed by our team (this cutting machine can slowly run and control cutting thickness), and a hole punch was used to determine its size in $8 \mathrm{~mm}$ diameter. After the adipose on surface of cancellous bone explant models was removed, these cancellous bone explant models were cultured with DMEM medium (containing 15\% FBS suitable for tissue cultivation) in the chamber of dynamic loading and circulating perfusion bioreactor system which has circulating perfusion effect during 3D cultivation besides mechanical load function.

\section{Micro-CT scanning and finite element analysis}

Mechanical stimulation can affect the proliferation and differentiation of bone cells, and ultimately affect regeneration of bone tissue, however, the strain and stress applying on cells in bone tissue cannot be measured accurately, but it can be effectively calculated in theory by Micro-CT scanning and finite element (FEM) analysis. In this experiment, the cancellous bone explant models extracted from rabbit femoral heads were scanned by a high-resolution Micro-CT (Skyscan 1076 X-ray Micro-tomography, 
Belgium) with a $9 \mu \mathrm{m}$ thickness in Beijing University of Aeronautics and Astronautics, China. Then the scanning results were treated with Mimics software for 3D models, three-dimensional inverse reconstruction software Geomagic for Nurbs surface, Solidworks for scaffold 3D model and FEM analysis in mechanical load of $1000 \mu \varepsilon$, $2000 \mu \varepsilon$ and $3000 \mu \varepsilon$ respectively.

\section{Alkaline phosphatase (AKP) activity assay}

After mechanical load using dynamic loading and circulating perfusion bioreactor system, all cancellous bone explant models were rinsed 3 times in PBS (unstressed model samples as the control group were incubated under the same conditions for the maximum period of mechanical loading application), these samples then were cut into about $1 \mathrm{~mm}^{3}$ size and tardily homogenized in RIPA buffer (400 $\left.\mu \mathrm{L}\right)$ containing protease and phosphatase inhibitors at $4^{\circ} \mathrm{C}$. Total protein was collected after centrifugation at $12000 \mathrm{r} / \mathrm{min}$ for $15 \mathrm{~min}$, and quantified by $\mathrm{BCA}^{\mathrm{TM}}$ Protein Quantification Kit. The absorbance (OD) value of AKP was detected according to AKP activity assay Kit, and its activity was calculated as the following formula:

$$
A K P(U / \text { gprot })=\frac{S O D}{S t O D} \times S t \text { phonel quantity }(0.003 \mathrm{mg}) \div S \text { protein quantity }(g) .
$$

SOD: Sample OD value; St OD: Standard substance OD value; St phonel quantity: Standard subatance phonel quantity; Sprotein quantity: Sample protein quantity.

\section{Tests of mechanical properties}

Mechanical properties of cancellous bone explant models were assessed on the classical mechanical Micro Tester (INSTRON 5865, USA). Testing conditions were set as a $0.5 \mathrm{~N}$ preload and $2 \mathrm{~mm} / \mathrm{min}$ loading rate until the occurrence of maximum stress/ maximum load, and a stress-strain, stress under maximum load and elastic modulus were obtained, which will determine the influence of different levels of mechanical load on the stiffness of bone explant models.

\section{Measurement of tissue mineral density}

Tissue mineral density (TMD) was determined by a high-resolution Micro-CT scanning described as above. The cancellous bone models were mounted in a cylindrical specimen holder to be captured in a single scan. Scanning conditions were set as a $55 \mathrm{kV}$ peak voltage and $9 \mu \mathrm{m}$ slices. Calculation of TMD $\left(\mathrm{g} / \mathrm{cm}^{3}\right)$ was performed according to gray value of bone explant models by the postprocessing of Micro-CT.

\section{Osteoid staining according to Von-kossa}

Originally designed as a technique to detect inorganic phosphates via silver nitrate, technique of Von-kossa has been found wide acceptance as a mineralized tissue marker. In this study, Von-kossa staining was performed to determine the presence of mineralization after rabbit cancellous bone explant models were stimulated with different mechanical loads. These models were fixed in $4 \%$ paraform for $24 \mathrm{~h}$ at room temperature, and made into $5 \mu \mathrm{m}$ undecalcified tissue sections (supported by Tianjin Hospital, China).The sections were de-hydrated and incubated with 5\% silver nitrate 
solution under ultraviolet light for $60 \mathrm{~min}$. Un-reacted silver was removed with distilled water for $5 \mathrm{~min}$ and $5 \%$ sodium thiosulfate for $2 \mathrm{~min}$. The latter was rinsed away for 5 min with distilled water again, and treated repeatedly by $0.1 \%$ nuclear fast red staining for $2 \mathrm{~min}$. Then all images were captured using a microscope (Olympus, Japan) with predetermined magnification of 10 and 20. To assess volume of osteoid, the osteoid bands were measured manually using Image Proplus 6.0. There were totally 5 samples in each group, and 8 fields of every sample were evaluated by this software.

\section{Observation of the fluorochrome double-labeling}

In fluorochrome labeling, two different substances were added to the culture medium in chamber of the dynamic loading and circulating perfusion bioreactor system at defined time: a dose of $5 \times 10^{-4} \mathrm{~mol} / \mathrm{L}$ Tetracycline hydrochloride from the first day to the $6^{\text {th }}$ day, and $50 \mu \mathrm{g} / \mathrm{ml}$ Calcein from the $9^{\text {th }}$ day to $14^{\text {th }}$ day or the $16^{\text {th }}$ day to the $21^{\text {st }}$ day. In labeling groups for 14 days and 21 days, all these cancellous bone explant models were arranged in $1000 \mu \varepsilon$ for $14 \mathrm{~d}, 2000 \mu \varepsilon$ for $14 \mathrm{~d}, 1000 \mu \varepsilon$ for $21 \mathrm{~d}$ and 2000 $\mu \varepsilon$ for $21 \mathrm{~d}$ respectively. Especially, labeling for $21 \mathrm{~d}$ without mechanical load was set as the control group. Then all the bone explant models were undecalcified with a thickness of $10 \mu \mathrm{m}$ as described above. The image acquisition of entire section were captured at $390 \mathrm{~nm}$ for Tetracycline and $485 \mathrm{~nm}$ for calcein respectively using a laser scanning confocal fluorescence microscope (Perlin Elmer Ultra View Vox, UK) with a magnification of 40. The evaluation of bone formation were also finished by Image Proplus 6.0.

\section{Western blot and ELISA assays}

Total protein was extracted from cancellous bone explant models and quantified described as above and all protein samples were stored at $-80^{\circ} \mathrm{C}$. For investigating some differences in protein expression of bone explant models under different levels of mechanical load, Western blot assay and ELISA assay were employed to evaluate COL-1, OPG and BMP-2 respectively.

In Western blot assay [10], each group, a total of $40 \mathrm{mg}$ of protein was separated by SDSPAGE and blotted to a PVDF membrane. The membrane was blocked in TBST with $5 \%$ skim milk for $1 \mathrm{~h}$ and probed overnight at $4^{\circ} \mathrm{C}$ with appropriate Mouse AntiCollagen I antibody (1:1000 dilution). After washing in TBS, the membrane was incubated with HRP conjugated goat anti-IgG secondary antibody (1:1000 dilution) at $37^{\circ} \mathrm{C}$ for $1 \mathrm{~h}$. Washing in TBS again later, the blots in the membrane were developed by an ECL detection kit (Cwbiotech, China) for 5 min and exposed to Medical X-ray Film. In this process, (glyceraldehyde 3-phosphate dehydrogenase) GAPDH was used as an internal reference control. Scion Image was used to perform semi-quantitative analysis.

ELISA of OPG and BMP-2, assays were performed according to the protocol of manufacturer using a specific ELISA kit. Each protein sample was conducted in triplicate with parallel 3-well culture plates to ensure accurate results. Then by using a professional software Curve Exert 1.3 provided by Cusabio Biotech Co., LTD, USA, a standard curve was made for calculation of OPG and Bmp-2 values.

\section{RNA extraction and quantitative real-time PCR}

Total RNA was extracted from the cancellous bone explant models with the Trizol reagent according to the manufacturer's instructions. Concentration and purity of RNA 
were determined by OD 260/280 nm absorption ratio. The total RNA was reversely transcribed into single-stranded cDNA using SuperRT cDNA Kit (Invitrogen), which were performed in a $20 \mu \mathrm{L}$ reaction mixture containing $500 \mu \mathrm{M}$ of dNTP Mix, $2 \mu \mathrm{l}$ of primer mix (Life Technologies, USA) and 200 units of Superscript III reverse transcriptase according to the manufacturer's instructions (Cwbiotech, BEI JING). The reaction mixture was incubated at $42^{\circ} \mathrm{C}$ for $60 \mathrm{~min}$ and at $85^{\circ} \mathrm{C}$ for $5 \mathrm{~min}$. Quantitative real-time PCR analysis was performed with an ABI 7500 fast Real-Time PCR machine (Applied Biosystems, Foster City, CA, USA) using a Fast SYBR-green Master Mix kit (Life Technologies, USA). The cycling profiles were $95^{\circ} \mathrm{C}$ for $20 \mathrm{~s}, 95^{\circ} \mathrm{C}$ for $3 \mathrm{~s}$ and $60^{\circ} \mathrm{C}$ for $30 \mathrm{~s}$ for a total of 40 cycles. The details of the primers were listed in Table 1. Three independent experiments were carried out to determine relative mRNA levels. Using the relative quantitative method, expression levels of PCR products were calculated.

\section{Statistical analysis}

All statistical analyses were performed using SPSS 13.0. All data were presented as the means \pm S.D. from at least three separate experiments with triplicate samples. Significant differences were evaluated by a two-tailed $t$ test. Significance was defined at $p<0.05$.

\section{Results}

\section{Culture and FEM analysis of bone explant models}

The cancellous bone explant models prepared from rabbit femoral heads under sterile conditions were of complete trabecular structure with a round and parallel shape $3 \mathrm{~mm}$ in thickness and $8 \mathrm{~mm}$ in diameter (Figure 1A and B). These models could be cultured in vitro using the dynamic loading and circulating perfusion bioreactor system for mechanical load study (Figure 1C and 1D) and also be scanned by Micro-CT for FEM analysis.

By Micro-CT scanning, a distinct structure and rich lacuna of trabecular bone could be observed in bone explant models, it is suitable for cultivation in vitro by circulating flow (Figure 2A); And a 3D model reconstructed by Mimics and scaffold 3D model are shown in Figure 2B, C, D and E. There were some parameters involved by Ansys12.0 to be shown in Table 2. In this analysis process, the number of elements affected by stress was assessed through indexes of $<500 \mu \varepsilon, 1000 \mu \varepsilon, 2000 \mu \varepsilon, 3000 \mu \varepsilon$ and $>4000 \mu \varepsilon$ (Table 3, Figure 2F, $\mathrm{G}$ and $\mathrm{H})$. These results showed that the number of elements was significantly increased when mechanical load was $3000 \mu \varepsilon$. According to the bone function adaptability model with strain set up by Frost, it might be considered bone physiological strain in 50-2500 $\mu \varepsilon$, because in the range it had a equal rate between bone formation and bone resorption,

Table 1 Sequences of primers used for qRT-PCR

\begin{tabular}{|c|c|c|}
\hline Gene name & Length (bp) & Sequences of primer \\
\hline \multirow{2}{*}{ BMP-2 } & \multirow{2}{*}{186} & 5'-GCGGTGGACTGCACAGGGAC-3' \\
\hline & & 3'-AGGGGGTGCCCCTTCCCATC-5' \\
\hline \multirow{2}{*}{ COL-1 } & \multirow{2}{*}{184} & 5'-CACATGCGTGCAGAACGGCG-3' \\
\hline & & $\overline{\text { 3'-CGCGTCTTCGGGGCAGACAG-5' }}$ \\
\hline \multirow{2}{*}{ OPG } & \multirow{2}{*}{211} & 5'-GCTTCGACGTCACCCCTGCC-3' \\
\hline & & 3'-AGGGGGTGCCCCTTCCCATC-5' \\
\hline \multirow{2}{*}{$\beta$-actin } & \multirow{2}{*}{295} & 5'-TGGCTCTAACAGTCCGCCTAG-3' \\
\hline & & 3'-AGTGCGACGTGGACATCCG-5' \\
\hline
\end{tabular}




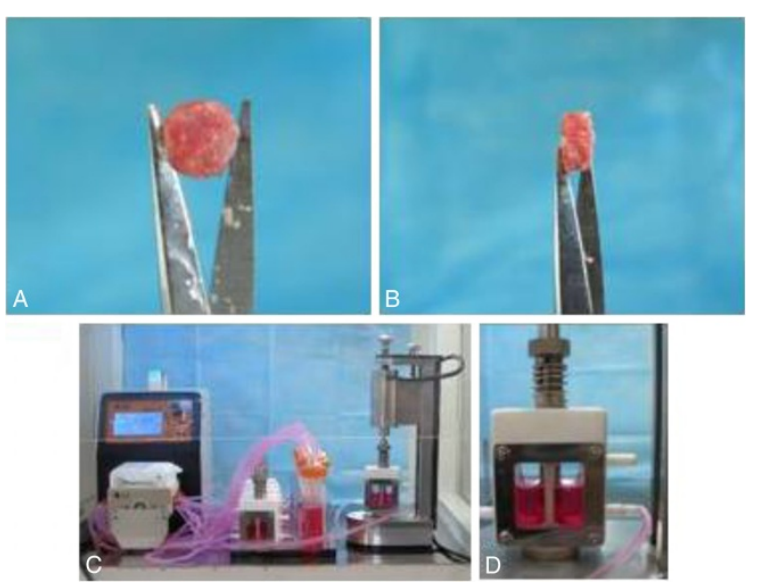

Figure 1 Cancellous bone explant models and bioreactor system for $3 \mathrm{D}$-cultivation. These models made from the rabbit femoral head were $8 \mathrm{~mm}$ in diameter and $3 \mathrm{~mm}$ in thickness, front (A) and side (B) view. the dynamic loading and circulating-perfusion bioreactor under working condition is shown in (C), and the chamber of the dynamic load and circulating-perfusion bioreactor system is presented in (D).
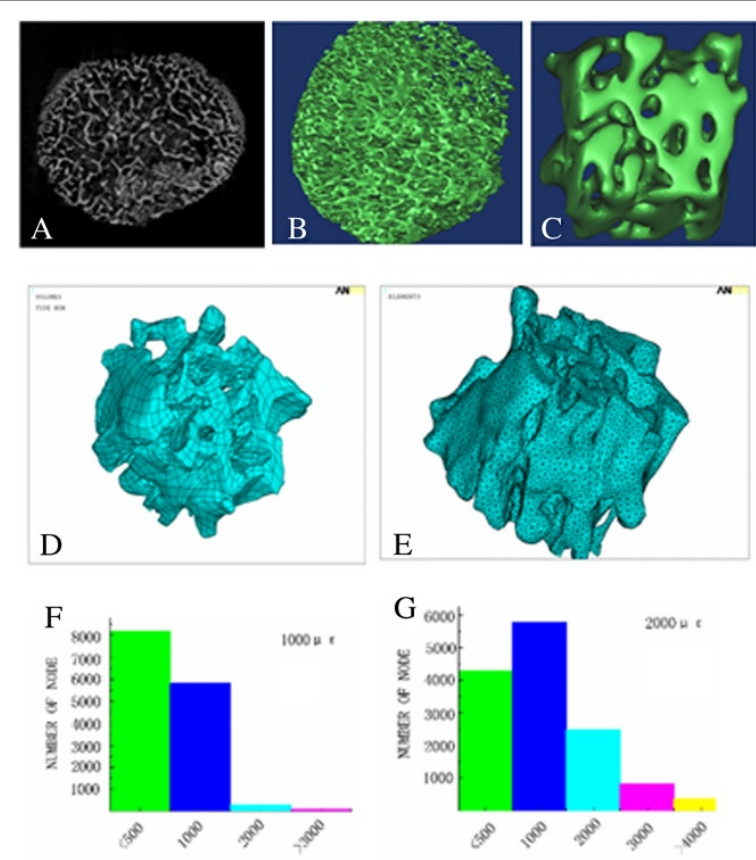

$\operatorname{sraux}(c)$

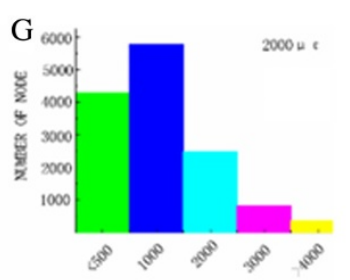

$\operatorname{sikaIx}(c)$

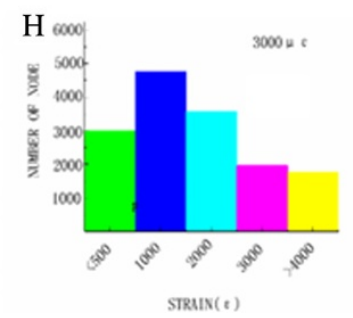

Figure 2 Results of Micro-CT scanning and FEM analysis. By Micro-CT scanning, microstructures of the explant models could be observed in (A). 3D model was generated in Mimics as showed in B (solid model) and $\mathbf{C}$ (free meshed). Using Solidworks, 3D model of the scaffold could be generated from these elements (D and E). By finite element (FEM) analysis, strain distribution results of group $1000 \mu \varepsilon, 2000 \mu \varepsilon$ and $3000 \mu \varepsilon$ were shown respectively in $\mathbf{F}, \mathbf{G}$ and $\mathbf{H}$ 
Table 2 Some parameters involved in finite element analysis by Ansys 12.0

\begin{tabular}{cccccc}
\hline $\begin{array}{c}\text { Element } \\
\text { type }\end{array}$ & $\begin{array}{c}\text { Young's modulus } \\
(\mathbf{M P a})\end{array}$ & $\begin{array}{c}\text { Poisson's } \\
\text { ratio }(\boldsymbol{v})\end{array}$ & $\begin{array}{c}\text { Number of } \\
\text { elements }\end{array}$ & Appearant strain $(\boldsymbol{\mu} \varepsilon)$ & Height $(\mathbf{m m})$ \\
\hline 10 node92 & 51.53 & 0.3 & 184035 & $1000-3000$ & 0.95 \\
\hline
\end{tabular}

Table 3 Strain distribution analysis of models under mechanical stimulus

\begin{tabular}{lcccc}
\hline \multirow{2}{*}{ Groups } & \multicolumn{4}{c}{ Numbers of elements in different strain ranges } \\
\cline { 2 - 5 } & $<\mathbf{5 0 0} \boldsymbol{\mu \varepsilon}$ & $\mathbf{1 0 0 0 - 3 0 0 0 ~} \boldsymbol{\mu \varepsilon}$ & $>\mathbf{3 0 0 0} \boldsymbol{\mu \varepsilon}$ & $>\mathbf{4 0 0 0} \boldsymbol{\mu \varepsilon}$ \\
\hline $1000 \mu \varepsilon$ & 8200 & 6000 & 100 & 400 \\
\hline $2000 \mu \varepsilon$ & 4400 & 9500 & & 1600 \\
\hline $3000 \mu \varepsilon$ & 2900 & 9800 & & \\
\hline
\end{tabular}

but mechanical load with larger than $3000 \mu \varepsilon$ would lead to pathological bone modeling and reconstruction, it was overload [16,17]. In our previous studies, with four point bending device in two-dimension condition, mechanical stimulation of $2500 \mu \varepsilon$ could promote the proliferation and differentiation of osteoblasts, and a damage would occur to osteoblasts in $4000 \mu \varepsilon$ or $5000 \mu \varepsilon$ [2,9]. In two-dimension condition, the mechanical stress on cells was able to be controlled, however, the mechanical stress was easily scattered in $3 \mathrm{D}$ bone tissue model. Therefore, we firstly selected mechanical load level of $1000 \mu \varepsilon, 2000$ $\mu \varepsilon, 3000 \mu \varepsilon$ and $4000 \mu \varepsilon$ in the following AKP detecting assay.

\section{Specific AKP activity}

In this assay, the cancellous bone explant models cultured in vitro experienced different AKP activities which were related to the mechanical load level. Compared with the control group, mechanical load of $3000 \mu \varepsilon$ and $4000 \mu \varepsilon$ at $1 \mathrm{~Hz}$ for $30 \mathrm{~min}$ per day in 5 days could significantly downgrade the AKP activity $(P<0.05)$. Thus, we have verified that overloading mechanical stress could occur phenomenon of bone absorption discussed in other paper. However, when models were treated with mechanical load of $1000 \mu \varepsilon$ and $2000 \mu \varepsilon$ at the same frequency and loading time per day for 14 days, there was a notable increase in AKP activity comparing to control group (Table 4). Considering the critical role of AKP activity in osteoblasts calcification [18] and its effect in this assay, we specially assessed whether load with different levels of $1000 \mu \varepsilon$ and $2000 \mu \varepsilon$ were able to improve tissue volume of rabbit femoral head cancellous bone explant models by a series of experiments.

\section{Mechanical property assessment}

Being loaded at $1 \mathrm{~Hz}$ for 30 min per day in 14 and 21 days, mechanical properties of these cancellous bone explants models were detected by INSTRON 5865 tester. In macroscopic view, the inspection showed different stress-strain curves among three

Table 4 Results of AKP activity assay of the explant models $(n=3)$

\begin{tabular}{llr}
\hline \multirow{2}{*}{ Groups } & \multicolumn{2}{c}{ AKP(U/gprot) } \\
\cline { 2 - 3 } & $\mathbf{5}$ days & $\mathbf{1 4}$ days \\
\hline Control & $27.350 \pm 0.071$ & $26.126 \pm 0.013$ \\
\hline $1000 \mu \varepsilon$ & $26.309 \pm 0.034$ & $29.181 \pm 0.041^{*}$ \\
\hline $2000 \mu \varepsilon$ & $28.121 \pm 0.212$ & $33.218 \pm 0.034^{*}$ \\
\hline $3000 \mu \varepsilon$ & $13.365 \pm 0.105$ & $11.151 \pm 0.108$ \\
\hline $4000 \mu \varepsilon$ & $10.161 \pm 0.121$ & $10.603 \pm 0.010$ \\
\hline${ }^{*} P<0.05$, compared with the control groups. & &
\end{tabular}




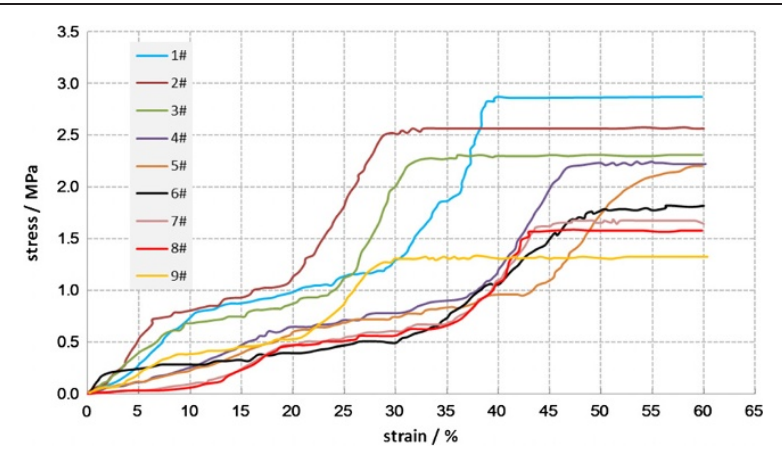

Figure 3 Stress-strain curves were obtained from mechanical tests of bone explant models on Instron 5865. Curves 1\#-3\# were from group $2000 \mu \varepsilon$, 4\#-6\# group $1000 \mu \varepsilon$ and 7\#-9\# the control group.

groups (Figure 3), further analysis indicated that, with increasing level of mechanical load, the elastic modulus and the stress under maximum load gradually increased, and mechanical strain of $2000 \mu \varepsilon$ for 21 days could markedly increase the elastic modulus of models, mechanical strain of $1000 \mu \varepsilon$ and $2000 \mu \varepsilon$ for 21 days had a significant effect on the stress of maximum load comparing to the control group $(\mathrm{P}<0.05$, Table 5$)$. Higher elastic modulus and stress of maximum load were apparent reflections of a better mechanical property of bones.

\section{Tissue mineral density analysis}

Like the mechanical property assay, the cancellous bone explant models were also treated with mechanical load of $1000 \mu \varepsilon$ and $2000 \mu \varepsilon$ for 14 and 21 days respectively. By scanning and analysis, an improvement was gradually seen in TMD with time increase under mechanical loading, especially, when cancellous bone explant models were treated with mechanical load of $1000 \mu \varepsilon$ and $2000 \mu \varepsilon$ for 21 days, there was an obvious difference in TMD comparing to control groups $(P<0.05$, Figure 4$)$. This analysis revealed that TMD variance could be related to the mechanical load level.

\section{Observation of Von-kossa and fluorochrome double labeling}

In order to determine whether mechanical load on cancellous bone explant models could influence osteoid formation in vitro, Von-kossa staining and fluorochrome double labeling were employed respectively at time points for 14 days and 21 days under lasting mechanical load. These assays demonstrated a presence of fresh osteoid within the cancellous bone explant models in all the mechanical load groups. In Von-kossa, the fresh osteoid is stained red and mineralized bone substance

Table 5 Mechanical property parameters of bone explant models $(n=3)$

\begin{tabular}{lccccc}
\hline Groups & \multicolumn{2}{c}{ Elastic modulus $(\mathbf{M P a})$} & & \multicolumn{2}{c}{ Stress of maximum load $(\mathbf{N})$} \\
\cline { 2 - 3 } \cline { 6 - 6 } & $\mathbf{1 4}$ days & $\mathbf{2 1}$ days & & $\mathbf{1 4}$ days & $\mathbf{2 1}$ days \\
\hline Control & $0.1107 \pm 0.0413$ & $0.1129 \pm 0.0344$ & & $51.8014 \pm 0.0182$ & $51.9311 \pm 0.0443$ \\
\hline $1000 \mu \varepsilon$ & $0.1134 \pm 0.0341$ & $0.1190 \pm 0.0243$ & & $51.9462 \pm 0.0733$ & $53.4947 \pm 0.1017^{*}$ \\
\hline $2000 \mu \varepsilon$ & $0.1503 \pm 0.0427$ & $0.1712 \pm 0.0125^{*}$ & & $51.9981 \pm 0.0655$ & $53.7294 \pm 2.3773^{*}$ \\
\hline${ }^{*} P<0.05$, compared with the control group. & & &
\end{tabular}




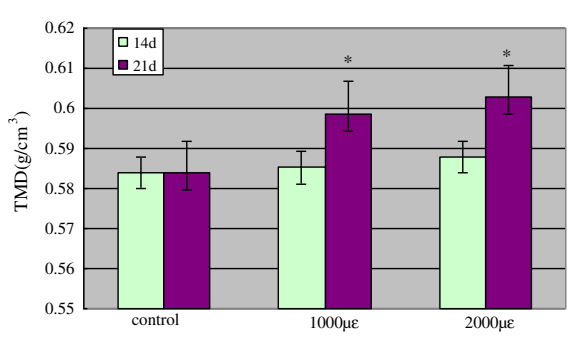

Figure 4 Tissue mineral density (TMD) analysis were performed using SPSS13.0. These analysis came respectively from the data of different cancellous bone model samples scanned by micro- $C T$ after being stimulated for 14 days and 21 days. In group $1000 \mu \varepsilon$ and $2000 \mu \varepsilon$ for 21 days group, the TMD had a change compared to the control group. ( ${ }^{*}<<0.05$ vs. the control group).

which is black (Figure 5), and in fluorochrome double labeling, the fresh osteoid were labelled by Tetracycline hydrochloride and Calcein (Figure 6). In every group, 40 fields was evaluated by analysis of Image Proplus 6.0. Arithmetic means were then calculated as measurements of collection. The effects of von-kossa and fluorochrome double labeling were compared in Figure 7 and Table 6. The analysis indicated that the osteoid formation were improved with the increasing of load intensity and time, the highest degrees of osteoid formation were seen in the group with maximum load of 2000 for $21 \mathrm{~d}$ in the two assays, but it was unparalleled between two assays. In Vonkossa, significant differences might be observed between the control group and the load groups, however, in fluorochrome double labeling, the significant differences were only found between control group and the latter three groups $(P<0.05)$.

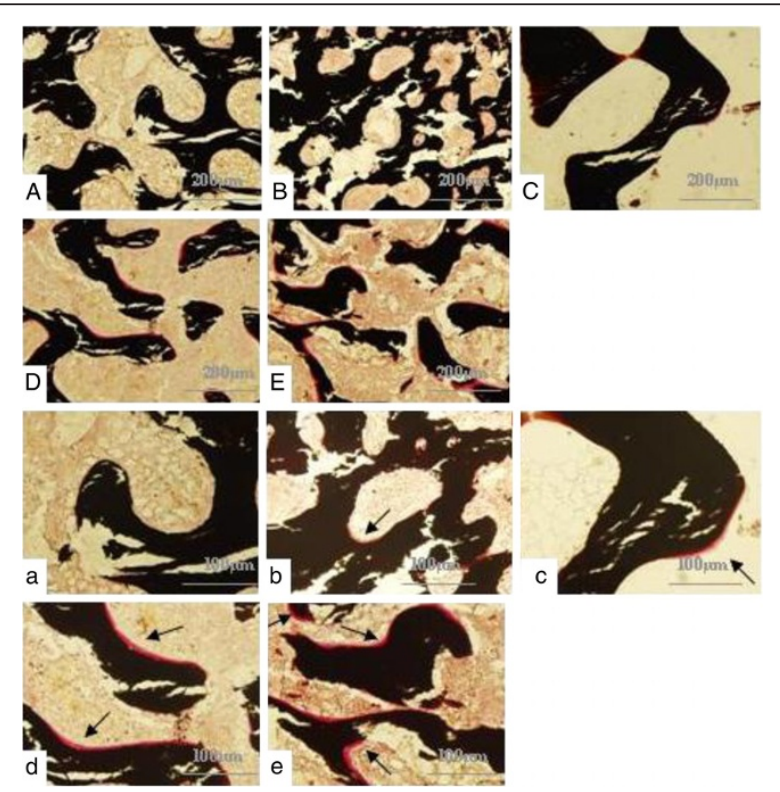

Figure $\mathbf{5}$ Results of Von-kossa staining. These photos were captured with predetermined light intensity under magnification of $10(\mathbf{A}-\mathbf{E})$ and $20(\mathbf{a}-\mathbf{e})$. Photos from $\mathbf{A}$ (or a) to $\mathbf{E}$ (or e) represented the control group, group $1000 \mu \varepsilon$ loading for 14 days, group $2000 \mu \varepsilon$ for 14 days, group $1000 \mu \varepsilon$ for 21 days and group 2000 $\mu \varepsilon$ for 21 days, respectively. The fresh osteoid have also been specially marked with arrow. 

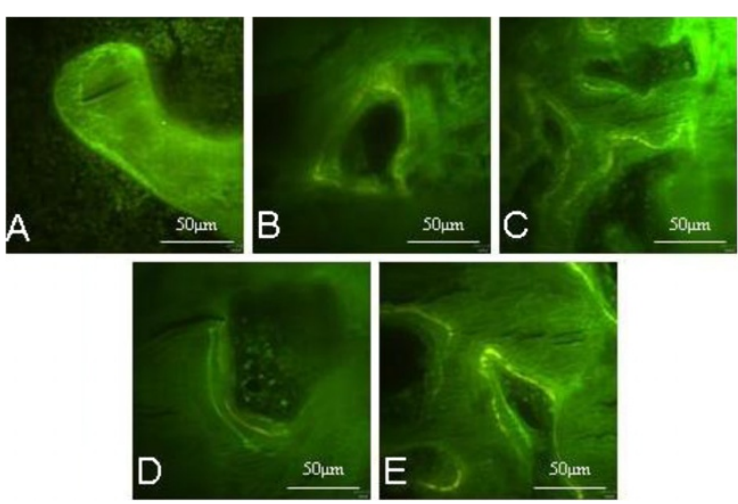

Figure 6 Results of fluorochrome double labeling. The explant models of cancellous bone were treated with Tetracycline hydrochloride in the first 6 days, then Calcein from the $9^{\text {th }}$ to the $14^{\text {th }}$ day and the $16^{\text {th }}$ to the $21^{\text {st }}$ day. The fresh osteoid were labelled in green or yellow by Tetracycline hydrochloride and Calcein in $\mathbf{A}$ (the control group cultured for 21 days), B (group $1000 \mu \varepsilon$ loading for 14days), C (group $2000 \mu \varepsilon$ loading for 14 days), $\mathbf{D}$ (group $1000 \mu \varepsilon$ loading for 21 days) and $\mathbf{E}$ (group $2000 \mu \varepsilon$ loading for 21 days).

\section{COL-1, OPG and BMP-2 protein expression}

COL-1, OPG and BMP-2 protein secreted by osteoblasts, play roles in regulation of bone formation and extracellular matrix. In the present study, these results indicated that mechanical load could regulate expression of COL-1, OPG and BMP-2 protein (Figure 8, Table 7). As showed in Figure 8 (2), expression values of COL-1 protein (relative to internal reference GADPH) in $1000 \mu \varepsilon$ and $2000 \mu \varepsilon$ for 21 days were significantly higher than the control group $(P<0.05)$ by western blot assay. Through ELISA assays, expression of OPG and BMP-2 protein were investigated. Interestingly, all expression values of OPG and BMP-2 were improved under mechanical load the comparing to control group $(P<0.05)$, yet which were not a striking action for expressing of OPG protein under mechanical load of $1000 \mu \varepsilon$ for 14 days.

\section{COL-1, OPG and BMP-2 mRNA expression}

The analysis results of Quantitative real-time PCR for the expression of COL-1, OPG and BMP-2 mRNA are summarized in Figure 9. We found that expression of three genes were gradually increased with improvement of mechanical load level at any time. Furthermore, under mechanical load of $1000 \mu \varepsilon$ and $2000 \mu \varepsilon$ for 21 days, there were

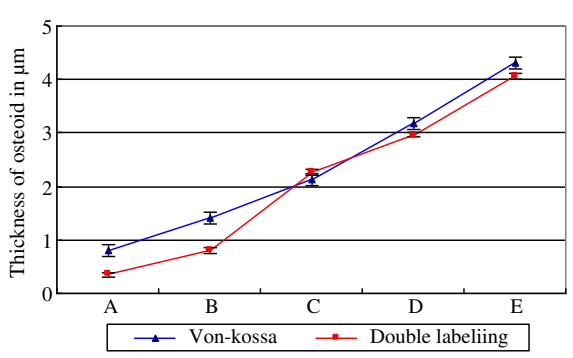

Figure 7 Osteoid formation of bone explant models. These analysis were conducted by Image Proplus 6.0 and shown as $\mathbf{A}$ (the control group cultured for 21 days), B (group $1000 \mu \varepsilon$ loading for 14 days), $\mathbf{C}$ (group $2000 \mu \varepsilon$ loading for 14 days), D (group $1000 \mu \varepsilon$ loading for 21 days) and E (group $2000 \mu \varepsilon$ loading for 21 days). 
Table 6 Osteoid formation of bone explant models $(n=40)$

\begin{tabular}{lll}
\hline \multicolumn{1}{c}{ Groups } & Von-kossa $(\boldsymbol{\mu m})$ & Double labeling $(\boldsymbol{\mu m})$ \\
\hline control & $0.8109 \pm 0.0013$ & $0.3491 \pm 0.0072$ \\
\hline $14 \mathrm{~d} 1000 \mu \varepsilon$ & $1.4024 \pm 0.0219^{*}$ & $0.7984 \pm 0.0081$ \\
\hline $14 \mathrm{~d} 2000 \mu \varepsilon$ & $2.1175 \pm 0.1129^{*}$ & $2.2718 \pm 0.0481^{*}$ \\
\hline $21 \mathrm{~d} 1000 \mu \varepsilon$ & $3.183 \pm 0.0876^{*}$ & $2.9632 \pm 0.0875^{*}$ \\
\hline $21 \mathrm{~d} 2000 \mu \varepsilon$ & $4.3038 \pm 0.0602^{*}$ & $4.0562 \pm 0.1027^{*}$ \\
\hline${ }^{*} P<0.05$, compared with the control group. & &
\end{tabular}

significant upregulation in three genes, but, to mechanical load for 14 days, the striking upregulation was only seen in COL-1 and BMP-2 under mechanical load of $2000 \mu \varepsilon$. In addition, compared with protein expression of OPG, COL-1 and BMP-2, it also revealed that the three proteins and the three genes could not accordantly response to the same mechanical load condition in vitro.

\section{Discussion}

During evolution, bones have optimized its load-bearing role by adapting its architecture and function to mechanical forces. Removal of mechanical load results in bone mass decrease while a suitable dynamic mechanical load can promote bone formation [19]. However, bones are sensitive to not static but dynamic load, static load has no effect on bone remodeling, whereas a similar dynamic load is associated with bone mass increase [20]. A single period of dynamic load can not only induce the periosteal surface to transform directly from quiescence to active bone formation [21], but also modulate bone loss caused by calcium insufficiency [22]. Therefore, dynamic mechanical load is a fundamental physiological factor for regulating bony structure and function of bones [23]. In the present study, we investigated the association between growth of rabbit cancellous bone explant models and mechanical load. Mechanical load conditions of rabbit cancellous bone explant models were obtained from finite element analysis. On the other hand, the adaptive ability of bone tissues to the mechanical environment depends on the bone cells [24]. Osteoblasts, the bone-forming cells are located on the surface of bones, which can be activated by dynamic mechanical stimulus in vitro. AKP is a differentiation marker of osteoblasts whose expression was enhanced under mechanical load of $1000 \mu \varepsilon$ and 2000 $\mu \varepsilon$ in our experiments, and thus, it was considered that osteoblasts in these cancellous

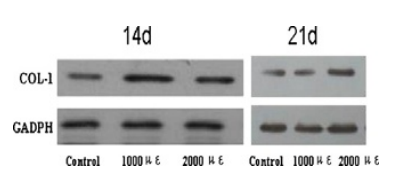

(1)

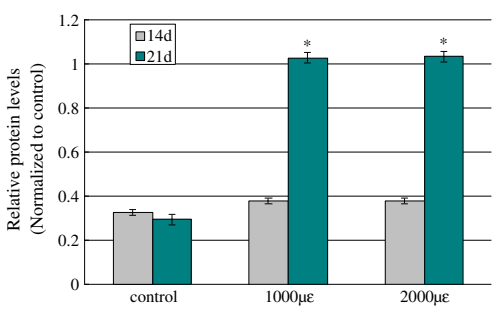

(2)

Figure 8 COL-1 protein expressing effect of mechanical load on bone explant models. (1) the explant samples of cancellous bone were pretreated with different mechanical intensity and time, by western-bloting, the expression of COL-1 and GADPH were identitied; (2) Through analysis of the gray values using Scion Image, COL-1 expressing variances were showed due to different stress levels, * $P<0.05$ compared with the groups. 
Table 7 OPG and BMP-2 effect of bone explant models $(n=3)$

\begin{tabular}{lllll}
\hline \multirow{2}{*}{ Groups } & $\mathbf{O P G}(\mathbf{p g} / \mathbf{m l})$ & BMP-2(pg/ml) & & \\
\cline { 2 - 4 } & $\mathbf{1 4}$ days & $\mathbf{2 1}$ days & $\mathbf{1 4}$ days & 21 days \\
\hline Control & $7.0490 \pm 0.126$ & $7.0553 \pm 0.219$ & $3.3844 \pm 0.227$ & $3.3391 \pm 0.391$ \\
\hline $1000 \mu \varepsilon$ & $8.7778 \pm 0.230$ & $15.3220 \pm 0.556^{*}$ & $4.7232 \pm 0.243^{*}$ & $6.4394 \pm 0.480^{*}$ \\
\hline $2000 \mu \varepsilon$ & $10.1096 \pm 0.366^{*}$ & $12.0148 \pm 0.476^{*}$ & $5.2203 \pm 0.140^{*}$ & $6.4730 \pm 0.480^{*}$ \\
\hline${ }^{*} P<0.05$, compared with the control group. & & &
\end{tabular}

bone explant models could be sensitive to mechanical load from a new dynamic loading and circulating perfusion bioreactor system.

By the increasing activity of AKP, it was presumed that osteoblasts in cancellous bone explant models might be supplied with adequate nutrients and preserved vigorous vitality. This is supported by Dodd et al. [25] with observation of reduction in the number of viable osteocytes as a result of the absence of mechanical stress in vivo, which was, however, reversible after applying mechanical stress. In our investigation, the osteoblast functions measured by osteoid formation in bone explant models (Von-kossa staining and two fluorochromes labelling) were significantly improved in relation to the level and time of mechanical load. Moreover, some other indexes on these models, such as TMD, stiffness and elastic modulus, were also measured with improvement accordingly for the occurrence of osteoid formation.

In addition, we also found a close association between COL-1, OPG and BMP-2 expression and mechanical load on cancellous bone explant models in this research. Previous studies have revealed that Osteogenic differentiation procedurally experiences gene expression of ALP, OPG, COL-1, and BMP-2 in a time-dependent manner. COL1 is the most abundant protein in bone and the main composition of bone matrix, its expression is complexly regulated by a set of different factors. Under 3D dynamic load condition, COL-1 could be up-regulated after 3 days. OPG secreted by osteoblasts is a sort of glycoprotein which can combine to osteoclast surface-factor NF- $\kappa \beta$ receptor activator (RANK) competing with OPGL which is cognate ligand of OPG, both OPG and OPGL can highly express in osteoblasts. RANK combining with OPG can block differentiation and proliferation of osteoclasts, which will reduce generation of bone absorption, additionally, osteoclast surface F-actin which is bound to OPG can directly inhibit bone resorption activity of mature osteoclasts; and BMP-2 plays an important role in the regulation of bone formation and remodeling, which can improve bone formation in bone tissue engineering, since all of them are secreted during differentiation or proliferation of osteoblasts [26-34]. Expressions of COL-1, OPG and BMP-2 protein and
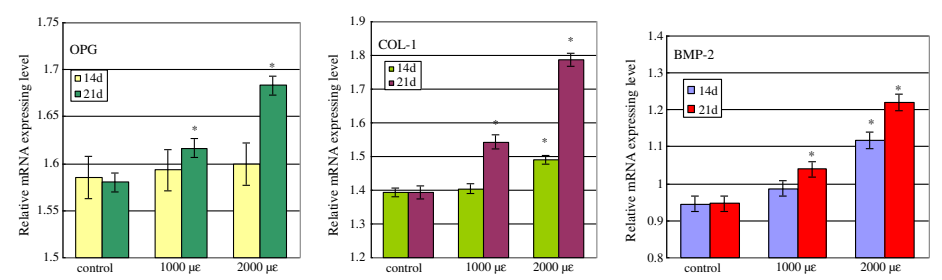

Figure 9 Expressions of OPG, COL-1 and BMP-2 mRNA were detected using real-time PCR after mechanical load with different intensities on the explant models for 14 days and 21 days. Mean $\mathrm{Ct}$ value of target genes was normalized to housekeeping gene $\beta$-actin. ${ }^{*} p<0.05$ compared with the control group. 
mRNA were assessed after mechanical load on cancellous bone explant models in our experiments, showing a significant increase in mechanical level and time dependent manner. It was made further clearly that these cancellous bone explant models had trended to development of bone formation in molecule.

In summary, our study demonstrated that mechanical load could regulate function and activity of osteoblasts in cancellous bone explant models. Through pathways of COL-1, OPG and BMP-2, mechanical load improved TMD, stiffness and elastic modulus due to the formation of fresh osteoid. This study firstly showed how mechanical load influenced development of rabbit cancellous bone explant models in microenvironment of dynamic loading and circulating perfusion bioreactor system.

\section{Abbreviations}

GFs: Growth factors; MAPK: Mitogen-activated protein kinase; ECM: Extracellular matrix; DMEM: Dulbecco's modified Eagle's medium; FBS: Fetal bovine serum; AKP: Alkaline Phosphatase; COL-1: Collagen I; OPG: Osteoprotegerin; Bmp2: Bone Morphogenetic Protein-2; PBS: Phosphate buffered saline; FEM: Finite element; OD: Optical density; TMD: Tissue mineral density.

\section{Competing interests}

The authors declare that they have no competing interests.

\section{Authors' contributions}

WZ and LR were responsible for conceiving and designing the study, preparation of models, and drafting the report. WZ and LL carried out the mechanical load assays, and performed the statistical analysis. LH was responsible for 3D models reconstruction and FEM analysis. $L$ and ZX carried out the molecular studies. GY were responsible for technical support during the mechanical load experiment. WZ and LJ have contributed equally to this work. ZX who is the Corresponding author revised the manuscript and provided the fee for these studies. All authors read and approved the final manuscript.

\section{Acknowledgments}

This study was supported by grants from the National Natural Science Foundation Key Project of China (10832012) and the National Natural Science Foundation Project of China (11072087).

\section{Author details}

${ }^{1}$ Institute of Medical Equipment, Academy of Military Medical Sciences, Tianjin, China. ${ }^{2}$ Department of Pharmacology, Logistics College of Chinese People's Armed Police Forces, Tianjin, China.

Received: 31 December 2012 Accepted: 15 April 2013

Published: 19 April 2013

\section{References}

1. Rubin C, Turner AS, Bain S, Mallinckrodt C, MC Leod K: Anabolism low mechanical signals strengthen long bones. Nature 2001, 412(6847):603-604.

2. Chen XY, Zhang XZ, Guo Y, Li RX, Lin JJ, Wei Y: The establishment of a mechanobiology model of bone and functional adaptation in response to mechanical loading. Clin Biomech 2008, 23(Suppl):s88-95.

3. Ma R, Zhu D, Gong H, Gu G, Huang X, Gao J, Zhang X: High-frequency and low-magnitude whole body vibration with rest days is more effective in improving skeletal micro-morphology and biomechanical properties in ovariectomised rodents. Hip Int 2012, 22(2):218-226.

4. Gong H, Zhu D, Gao J, Lv L, Zhang X: An adaptation model for trabecular bone at different mechanical levels. Biomed Eng Online 2010, 9:32. doi:10.1186/1475-925X-9-32.

5. Reijnders CM, Bravenboer N, Holzmann PJ, Bhoelan F, Blankenstein MA, Lips P: In vivo mechanical loading modulates insulin-like growth factor binding protein-2 gene expression in Rat osteocytes. Calcif Tissue Int 2007, 80(2):137-143.

6. Dibbets JMH: One century of Wolff's law. In Bone Dynamics in Orthodontic and Orthopaedic Treatment. Edited by Carlson DS, Goldstein SA. Ann Arbor: Center for Human Growth and Development,University of Michigan Press; 1992:1-13.

7. Frost HM: Bone"mass"and the"mechanostat": a pro-posal. Anat Rec 1987, 219(1):1-9.

8. Wolff J: Das Gesetz der Transformation der Knochen. Berlin: Kirschwald; 1892:110-157.

9. Wang L, Zhang XZ, Guo Y, Chen XZ, Li RX, Liu L, Shi CH, Guo C, Zhang Y: Involvement of BMPs/smad signaling pathway in mechanical response in osteoblasts. Cell Physiol Biochem 2010, 26:1093-1102.

10. Yan YX, Gong YW, Guo Y, LV Q, Guo C, Zhuang Y, Zhang Y, Li R, Zhang XZ: Mechanical strain regulates osteoblast proliferation through integrin-mediated ERK activation. PLOS One 2012, 7(4):e35709.

11. Rubin J, Rubin C, Jacobs CR: Molecular pathways mediating mechanical signaling in bone. Gene 2006, 367:1-16.

12. Harada S, Rodan GA: Control of osteoblast function and regulation of bone mass. Nature 2003, 423:349-55.

13. Jones DB, Broeckmann E, Pohl T, Smith EL: Development of a mechanical testing and loading system for trabecular bone studies for long-term culture. Eur Cell Mater 2003, 5:48-60.

14. Jaasma MJ, Plunkett NA, Fergal JO'B: Design and validation of a dynamic flow perfusion bioreactor for use with compliant tissue engineering scaffolds. J Biotechnol 2008, 133:490-496. 
15. Xuezhong CHEN, Caihong SHI, Ruixin LI, Yong G, Zhihong L, Liang W, Xizheng Z: Design of a new dynamic load and circulating-perfusion bioreactor System. Journal of Medical Biomechanics 2011, 26(5):441-447.

16. Frost HM: Why do marathon runners have less bone than weight lifters?A vital-biomechanical view and explanation. Bone 1997, 20(3):183-189.

17. Frost HM: The mechanostat:a proposed pathogenic mechanism of osteoporoses and the bone mass effects of mechanical and nonmechanical agents. Bone Miner 1987, 2(2):73-85.

18. Anderson HC: Mechanism of mineral formation in bone. Lab Invest 1989, 60:320-330.

19. Rubin CT, Lanyon LE: Reglulation of bone formation by appied dynamic loads. J Bone Joint Surg Am 1984, 66:397-402.

20. Lanyon LE, Rubin CT: Static versus dynamic loads as an influence on bone remodeling. J Biomech 1984, 17:897-905.

21. Pead MJ, Skerry TM, Lanyon JE: Direct transformation from quiescence to bone formation in the adult periosteum foolowing a singe brief period of bone loading. J Bone Miner Res 1988, 3:647-656.

22. Lanyon $L E$, Rubin $C T$, Baust $G$ : Modulation of bone loss during calcium insufficiency by controlled dynamic loading. Calcif Tissue Int 1986, 38:209-216.

23. Lanyon LE: Control of bone architecture by functional load bearing. J Bone Miner Res 1992, 7(Supp2):S369-375.

24. Turner $\mathrm{CH}$, Pavalko FM: Mechanotransduction and functional reponse of the skeleton to physical stress:the mechanisms and mechanics of bone adaptation. J Orthop Sci 1998, 3:346-355.

25. Dodd JS, Raleigh JA, Gross TS: Osteocyte hypoxia: a novel mechanotransduction pathway. Am J Physiol 1999, 277(3Pt.1):C598-602.

26. Yourek G, McCormick SM, Mao JJ, Reilly GC: Shear stress induces osteogenic differentiation of human mesenchymal stem cells. Regen Med 2010, 5(5):713-724.

27. Yanagisawa M, Suzuki N, Mitsui N, Koyama Y, Otsuka K, Shimizu N: Compressive force stimulates the expression of osteogenesis-related transcription factors in ROS 17/2.8 cells. Arch Oral Biol 2008, 53(3):214-219.

28. Haasper C, Jagodzinski M, Drescher M, Meller R, Wehmeier M, Krettek C, Hesse E: Cyclic strain induces FosB and initiates osteogenic differentiation of mesenchymal cells. Exp Toxicol Pathol 2008, 59(6):355-363.

29. Yamazaki M, Fukushima H, shin M: Tumor necrosis factor alpha represses bone morphogenetic protein (BMP) signaling by interfering with the DNA binding of Smads through the activation of NF-kappaB. J Biol Chem 2009, 284:35987-35995

30. Lieb E, Milz S, Vogel T, Hacker M, Dauner M, Schulz MB: Effects of transforming growth factor- $\beta 1$ on bonelike tissue formation in three-dimensional cell culture. Tissue Eng 2004, 10(9/10):1399-1413.

31. Schofer MD, Veltum A, Theisen C, Chen F, Agarwal S, Fuchs-Winkelmann S, Paletta JR: Functionalisation of PLLA nanofiber scaffolds using a possible cooperative effect between collagen type I and BMP-2: impact on growth and osteogenic differentiation of human mesenchymal stem cells. J Mater Sci Mater Med 2011, 22(7):1753-1762.

32. Stangenberg L, Schaefer DJ, Buettner O, Ohnolz J, Möbest D, Horch RE, Stark GB, Kneser U: Differentiation of osteoblasts in three-dimensional culture in processed cancellous bone matrix: quantitative analysis of gene expression based on real-time reverse transcription-polymerase chain reaction. Tissue Eng 2005, 11(5/6):855-864

33. Hakeda Y, Kobayashi Y, Yamaguchi H, Tsuda E, Higashio K, Miyata T, Kumegawa M: Osteoclastogenesis inhibitory factor (OCIF) directly inhibits bone-resorbing activity of isolated mature osteoclasts. B iochem Biophys Res Commun 1998, 251(3):796-801.

34. Kadow RA, Hoffmann JE, Duda G, Wildemann B, Schmidmaier G: Effect of mechanical stimulation on osteoblastand osteoclast-like cells in vitro. Cells Tissues Organs 2009, 190(2):61-68.

doi:10.1186/1475-925X-12-35

Cite this article as: Zong ming et al:: Bone formation in rabbit cancellous bone explant culture model is

enhanced by mechanical load. BioMedical Engineering OnLine 2013 12:35.

\section{Submit your next manuscript to BioMed Central and take full advantage of:}

- Convenient online submission

- Thorough peer review

- No space constraints or color figure charges

- Immediate publication on acceptance

- Inclusion in PubMed, CAS, Scopus and Google Scholar

- Research which is freely available for redistribution

Submit your manuscript at www.biomedcentral.com/submit 